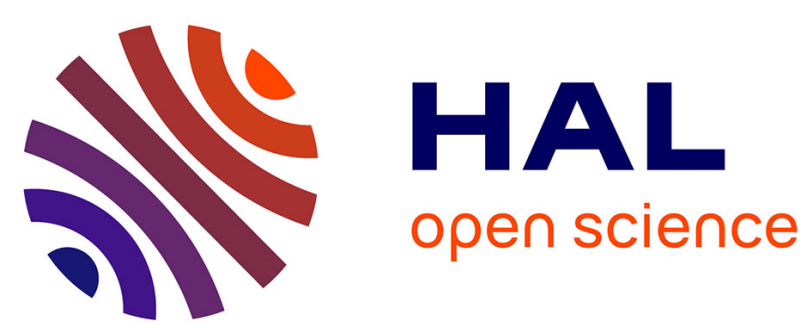

\title{
Vision-based Modeling and Control of Large-Dimension Cable-Driven Parallel Robots
}

Tej Dallej, Marc Gouttefarde, Nicolas Andreff, Redwan Dahmouche, Philippe

Martinet

\section{- To cite this version:}

Tej Dallej, Marc Gouttefarde, Nicolas Andreff, Redwan Dahmouche, Philippe Martinet. Vision-based Modeling and Control of Large-Dimension Cable-Driven Parallel Robots. IROS: Intelligent Robots and Systems, Oct 2012, Vilamoura, Algarve, Portugal. pp.1581-1586, 10.1109/IROS.2012.6385504 . lirmm-00737658

\section{HAL Id: lirmm-00737658 https://hal-lirmm.ccsd.cnrs.fr/lirmm-00737658}

Submitted on 14 Feb 2018

HAL is a multi-disciplinary open access archive for the deposit and dissemination of scientific research documents, whether they are published or not. The documents may come from teaching and research institutions in France or abroad, or from public or private research centers.
L'archive ouverte pluridisciplinaire HAL, est destinée au dépôt et à la diffusion de documents scientifiques de niveau recherche, publiés ou non, émanant des établissements d'enseignement et de recherche français ou étrangers, des laboratoires publics ou privés. 


\title{
Vision-based Modeling and Control of Large-Dimension Cable-Driven Parallel Robots
}

\author{
Tej Dallej *, Marc Gouttefarde ${ }^{\triangleright}$, Nicolas Andreff ${ }^{* \circ}$, Redwan Dahmouche * and Philippe Martinet ${ }^{* \boldsymbol{\Delta}}$ \\ Email: tej.dallejelasmea.univ-bpclermont.fr \\ http://ip.univ-bpclermont.fr
}

\begin{abstract}
This paper is dedicated to vision-based modeling and control of large-dimension parallel robots driven by inextensible cables of non-negligible mass. An instantaneous inverse kinematic model devoted to vision is introduced. This model relies on the specificities of a parabolic profile hefty cable modeling and on the resulting simplified static analysis. By means of a kinematic visual servoing method, computer vision is used in the feedback loop for easier control. According to the modeling derived in this paper, measurements that allow the implementation of this visual servoing method consist of the mobile platform pose, the directions of the tangents to the cable curves at their drawing points and the cable tensions. The proposed visual servoing scheme will be applied to the control of a large parallel robot driven by eight cables. To this end, in order to obtain the aforementioned desired measurements, we plan to use a multi-camera setup together with force sensors.
\end{abstract}

\section{INTRODUCTION}

Parallel cable-driven robots are a particular type of parallel kinematic machines in which cables connect the base to the mobile platform [1], [2]. A 6-DOF parallel robot driven by six cables can be thought as a Gough-Stewart platform turned upside down with cables instead of prismatic actuators. When the cables are tensed and considered massless and inextensible, the kinematic modeling of Gough-Stewart platforms and of parallel cable-driven robots are very similar.

Visual servoing techniques [3], [4], [5] were applied to parallel robotics [6], [7], [8]. These applications rely on 3D visual servoing where the mobile platform pose is indirectly measured and used for regulation. To make control robust with respect to modeling errors, it was proposed to servo the leg edges [9] which improved the practical robustness by servoing the legs in the image. This method has been validated on a broad class of parallel robots [10].

Using a 3D pose kinematic visual servoing method, in which the mobile platform pose is used for regulation, the work reported in [11] confirmed that visual servoing techniques are a good alternative for the control of parallel cable robots. However, in [11], the cables are supposed to be massless. This assumption is invalid in the case of largedimension cable robots [12] since the cables sag under their own weight. Assuming that the cable is inextensible and the

* Institut Pascal, UBP/CNRS/IFMA, Clermont-Ferrand, France.

$\triangleright$ LIRMM, CNRS/UM2, Montpellier, France.

- Institut FEMTO-ST, Univ. Franche-Comté/CNRS/ENSMM/UTBM, Besançon, France.

A IRCCYN, CNRS/INS2I/INSIS/INSB, Nantes Cedex, France.

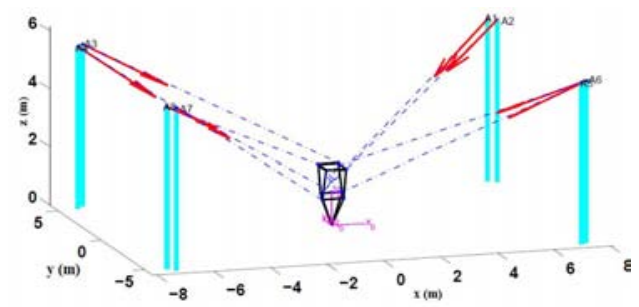

Fig. 1. Sketch of the LIRMM/Tecnalia CoGiRo robot

sag relatively small, a simplified hefty cable modeling can be considered [13]. This simplified modeling describes the cable profile explicitly as a parabolic curve. Compared to the use of the elastic catenary [14], it leads in turn to a simplified (quasi) static analysis of large-dimension parallel cable robots [15].

The contribution of this paper is to extend the vision-based control proposed in [11] to large-dimension parallel cable robots. To this end, based on the aforementioned simplified hefty cable modeling, the instantaneous inverse kinematic model of large-dimension parallel cable robots is determined. This model turns out to be dependent on the pose of the mobile platform but also on the directions of the tangents to the cables at their drawing points and on the cable tensions.

The cable tensions can be obtained by means of force sensors. To estimate the mobile platform pose and the cable tangent directions, one can rely on vision. In the case of a large-dimension cable robot, it is obviously not conceivable to measure all the needed variables using a single perspective camera in front of the robot. Instead, we plan to use a multicamera perception system. Firstly, several cameras observing visual targets attached to the mobile platform can provide an accurate pose estimation [16]. Using, e.g., four cameras provides a wide field of view and should always ensure the observation of significant part of the targets. Secondly, by locally observing each cable by means of a stereo pair (two cameras), 3D reconstruction yields the direction of the tangent to the cable at its drawing point. Indeed, a sagging cable can locally be approximated as a straight line segment.

This paper is organized as follows. Section II presents a simplified modeling of an inextensible cable of nonnegligible mass. In Section III, the corresponding instantaneous inverse kinematic model of large-dimension parallel cable robots is derived. Section IV is dedicated to the visionbased control strategy. The presented method is validated 
on the simulator of the parallel CoGiRo cable robot in Section V. Conclusions are finally drawn in Section VI.

\section{MODEL OF CABLE WITH NON-NEGLIGIBLE MASS DEVOTED TO VISION}

Throughout the paper, the notations given in Table I will be used.

- $i=1 \ldots k$ denotes the driving cables, where $k$ is the number of cables.

- Boldface characters denote vectors. Unit vectors are underlined.

- $\mathcal{F}_{A i}=\left(\mathbf{A}_{i}, \underline{\mathbf{x}}_{A i}, \underline{\mathbf{y}}_{A i}, \underline{\mathbf{z}}_{A i}\right), \mathcal{F}_{b}=\left(\mathbf{O}, \underline{\mathbf{x}}_{b}, \underline{\mathbf{y}}_{b}, \underline{\mathbf{z}}_{b}\right)$ and $\mathcal{F}_{e}=$ $\left(\mathbf{E}, \underline{\mathbf{x}}_{e}, \underline{\mathbf{y}}_{e}, \underline{\mathbf{z}}_{e}\right)$ denote the cable $i$, base and end-effector reference frames, respectively.

- $\mathcal{F}_{f}=\left(\mathbf{O}_{f}, \underline{\mathbf{x}}_{f}, \underline{\mathbf{y}}_{f}, \underline{\mathbf{z}}_{f}\right)$ is a fixed reference frame attached to the base of the robot. It can be a camera reference frame (eye-to-hand) or a pattern reference frame (eye-in-hand).

- $\mathcal{F}_{m}=\left(\mathbf{m}, \underline{\mathbf{x}}_{m}, \underline{\mathbf{y}}_{m}, \underline{\mathbf{z}}_{m}\right)$ is a mobile reference frame attached to the mobile platform.

- $\mathcal{F}_{p}=\left(\mathbf{O}_{p}, \underline{\mathbf{x}}_{p}, \underline{\mathbf{y}}_{p}, \underline{\mathbf{z}}_{p}\right)$ is the reference frame attached to the pattern.

- In the case of multiple cameras, $\mathcal{F}_{c j}=\left(\mathbf{O}_{c j}, \underline{\mathbf{x}}_{c j}, \underline{\mathbf{y}}_{c j}, \underline{\mathbf{z}}_{c j}\right)$ is the reference frame attached to the camera $j, j=1 \ldots r, r$ is the number of cameras.

- ${ }^{j} \mathbf{v}$ is vector $\mathbf{v}$ expressed in $\mathcal{F}_{j}$.

- $\mathcal{F}_{j *}$ is the desired position and orientation of $\mathcal{F}_{j}$.

- $g$ is the norm of the gravity acceleration vector.

- $q_{i}$ defines the motorized joint angle $i$.

- $\rho_{0}$ is the linear mass density of cable $i$.

- $L_{i}$ is the length of cable $i$ supposed to be massless (straight line segment).

- $l_{i}$ is the length of inextensible cable $i$ with non-negligible mass (with the parabolic profile modeling).

- $\mathbf{A}_{i}=\left(\begin{array}{lll}A_{i x} & A_{i y} & A_{i z}\end{array}\right)^{T}$ and $\mathbf{B}_{i}=\left(\begin{array}{lll}B_{i x} & B_{i y} & B_{i z}\end{array}\right)^{T}$ are the two extremities of the sagging part of cable $i$.

- $\underline{\mathbf{u}}_{i}=\left(\begin{array}{lll}u_{i x} & u_{i y} & u_{i z}\end{array}\right)^{T}$ is the unit vector pointing from $\overline{\mathbf{A}}_{i}$ to $\mathbf{B}_{i}$.

- $\vartheta_{\boldsymbol{A i}}=\vartheta_{A i} \underline{\mathbf{u}}_{A i}=\left(\begin{array}{lll}\vartheta_{A i x} & \vartheta_{A i y} & \vartheta_{A i z}\end{array}\right)^{T}$ is the force in the cable at point $\mathbf{A}_{i}$. The norm and direction of this force are the tension $\vartheta_{A i}$ and the unit vector $\underline{\mathbf{u}}_{A i}$, respectively.

- $\boldsymbol{\vartheta}_{B i}=\vartheta_{B i} \underline{\mathbf{u}}_{B i}=\left(\begin{array}{lll}\vartheta_{B i x} & \vartheta_{B i y} & \vartheta_{B i z}\end{array}\right)^{T}$ is the force in the cable at its attachment point $\mathbf{B}_{i}$. The norm and direction of this force are the tension $\vartheta_{B i}$ and the unit vector $\underline{\mathbf{u}}_{B i}$, respectively.

- ${ }^{i} \mathbf{T}_{j}=\left(\begin{array}{cc}{ }^{i} \mathbf{R}_{j} & { }^{i} \mathbf{t}_{j} \\ \mathbf{0} & \mathbf{1}\end{array}\right)$ is the homogeneous matrix associated to the rigid transformation from $\mathcal{F}_{i}$ to $\mathcal{F}_{j}$.

- ${ }^{j} \boldsymbol{\tau}_{i}=\left({ }^{j} \mathbf{V}_{i}{ }^{j} \boldsymbol{\Omega}_{i}\right){ }^{T}$ is the Cartesian velocity (linear and angular velocities) of the origin of $\mathcal{F}_{i}$ expressed in $\mathcal{F}_{j}$.

- $\mathbf{M}^{+}$is the pseudo-inverse of $\mathbf{M}$.

- $[\mathbf{a}]_{\times}$is the cross-product matrix associated with vector $\mathbf{a}$.

- $\widehat{\mathbf{M}}$ is the estimation of $\mathbf{M}$ based on measurements.

\section{TABLE I}

NOTATIONS USED THROUGHOUT THE PAPER

Fig. 2 shows the profile of a cable with non-negligible mass. In static loading conditions, it lies in the vertical plane $\Pi_{i}$ containing $\mathbf{A}_{i}$ and $\mathbf{B}_{i}$. The reference frame $\mathcal{F}_{A i}$ attached to $\Pi_{i}$ is obtained from frame $\mathcal{F}_{b}$ by a rotation of angle $\gamma_{i}$ around the $z$ axis $\left(\underline{\mathbf{z}}_{b}=\underline{\mathbf{z}}_{A i}\right.$ being vertical).

Given ${ }^{b} \underline{\mathbf{u}}_{i}=\left({ }^{b} u_{i x}{ }^{b} u_{i y}{ }^{b} u_{i z}\right)^{T}$, the angle $\gamma_{i}$ can be computed as:

$$
\gamma_{i}=\tan ^{-1}\left(\frac{{ }^{b} u_{i y}}{{ }^{b} u_{i x}}\right)
$$

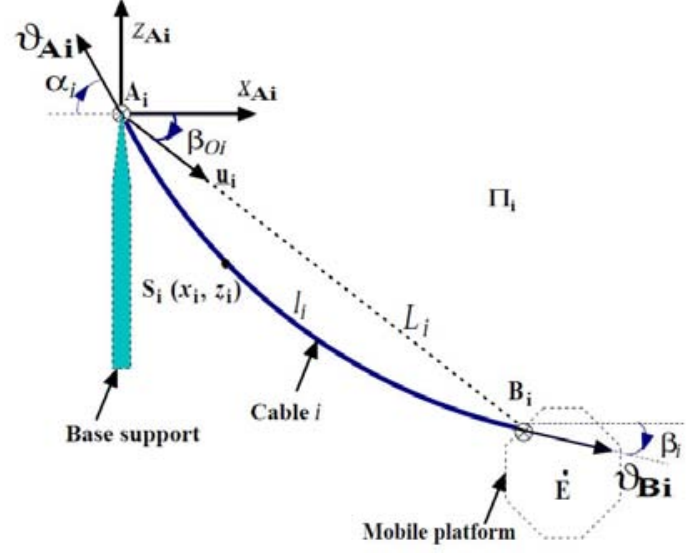

Fig. 2. Static equilibrium of cable $i$, with non-negligible mass.

A simplified modeling of an inextensible hefty cable [13] is used in this paper. This model is obtained from the elastic catenary modeling [14], [15]. It results in a parabolic cable profile equation, expressed in frame $\mathcal{F}_{A i}$, which is given by:

$$
z_{i}=-\frac{\rho_{0} g L_{i}}{2^{A i} \vartheta_{B i x}{ }^{A i} B_{i x}} x_{i}\left({ }^{A i} B_{i x}-x_{i}\right)+x_{i} \tan \left(\beta_{0 i}\right)
$$

where $x_{i}$ and $z_{i}$ are the coordinates in frame $\mathcal{F}_{A i}$ of cable point $\mathbf{S}_{i}$ (see Fig. 2).

The angle $\beta_{0 i}$ between $\underline{\mathbf{u}}_{i}$ and $\underline{\mathbf{x}}_{A i}$ (Fig. 2) can be written as:

$$
\beta_{0 i}=\tan ^{-1}\left(\frac{{ }^{A i} B_{i z}}{{ }^{A i} B_{i x}}\right)
$$

\section{A. Tangents to the cable profile}

The slope of the tangents to the cable profile (Eq. (2)) at points $\mathbf{B}_{i}$ and $\mathbf{A}_{i}$ (see Fig. 2) can be computed using ${\frac{d z_{i}}{d x_{i}}\left(x_{i}=B_{i x}\right)}=\tan \left(\beta_{i}\right)$ and $\frac{d z_{i}}{d x_{i}\left(x_{i}=0\right)}=\tan \left(\alpha_{i}\right)$, respectively.

According to (2), one can write:

$$
\left\{\begin{array}{l}
\tan \left(\beta_{i}\right)=\frac{{ }^{A i} \vartheta_{B i z}}{A i \vartheta_{B i x}}=\tan \left(\beta_{0 i}\right)+\frac{\rho_{0} g L_{i}}{2^{A i} \vartheta_{B i x}} \\
\tan \left(\alpha_{i}\right)=\frac{A i \vartheta_{A i z}}{{ }^{i i} \vartheta_{A i x}}=\tan \left(\beta_{0 i}\right)-\frac{\rho_{0} g L_{i}}{2^{A i} \vartheta_{B i x}}
\end{array}\right.
$$

Since ${ }^{A i} \vartheta_{A i x}=-{ }^{A i} \vartheta_{B i x}$ (as the only external loading applied to the cable between $\mathbf{A}_{i}$ and $\mathbf{B}_{i}$ is the cable weight) and introducing ${ }^{A i} \underline{\mathbf{u}}_{i}=\left(\begin{array}{ccc}\cos \left(\beta_{0 i}\right) & 0 & \sin \left(\beta_{0 i}\right)\end{array}\right)^{T}$, one can compute:

$$
\left\{\begin{array}{l}
{ }^{A i} \boldsymbol{\vartheta}_{B i}=\frac{{ }^{A i} \vartheta_{B i x}}{\cos \left(\beta_{0 i}\right)}{ }^{A i} \underline{\mathbf{u}}_{i}+\frac{\rho_{0} g L_{i}}{2} A i \\
{ }^{A i} \underline{\mathbf{z}}_{A i} \\
{ }^{A i}=-\frac{{ }^{i} \vartheta_{B i x}}{\cos \left(\beta_{0 i}\right)}{ }^{A i} \underline{\mathbf{u}}_{i}+\frac{\rho_{0} g L_{i}}{2}{ }^{A i} \underline{\mathbf{z}}_{A i}
\end{array}\right.
$$

It is important to note that the last equation can be expressed in any Euclidean reference frame. In the general case, we can write:

$$
\left\{\begin{array}{l}
\boldsymbol{\vartheta}_{\boldsymbol{B i}}=\mathbf{u}_{B i}=\mathbf{u}_{i}+\mathbf{u}_{\rho_{i}} \\
\boldsymbol{\vartheta}_{\boldsymbol{A i}}=\mathbf{u}_{A i}=-\mathbf{u}_{i}+\mathbf{u}_{\rho_{i}}
\end{array}\right.
$$

where $\mathbf{u}_{i}=\frac{{ }^{A i} \vartheta_{B i x}}{\cos \left(\beta_{0 i}\right)} \underline{\mathbf{u}}_{i}, \mathbf{u}_{\rho_{i}}=\frac{\rho_{0} g L_{i}}{2} \underline{\mathbf{Z}}_{A i}$. 


\section{B. Inverse kinematics}

The inverse kinematics gives the length of each cable for a given pose of the platform. Using the cable profile of Eq. (2), one can compute the cable length by integrating a cable length element [15]:

$$
\begin{aligned}
l_{i} & =\int_{0}^{A i} B_{i x} \sqrt{1+\left(\frac{d z}{d x}\right)^{2}} \mathrm{~d} x \\
& ={ }^{A i} B_{i x} \frac{c_{1 i} k_{1 i}-c_{2 i} k_{2 i}+\ln \left(\frac{c_{1 i}+k_{1 i}}{c_{2 i}+k_{2 i}}\right)}{2 r_{i}}
\end{aligned}
$$

where $r_{i}=\frac{\rho_{0} g L_{i}}{A i \vartheta_{B i x}}, k_{1 i}=\tan \left(\beta_{0 i}\right)+\frac{r_{i}}{2}, k_{2 i}=\tan \left(\beta_{0 i}\right)-\frac{r_{i}}{2}$, $c_{1 i}=\sqrt{1+k_{1 i}^{2}}$ and $c_{2 i}=\sqrt{1+k_{2 i}^{2}}$.

\section{VISION-BASED KINEMATICS}

The instantaneous inverse kinematic model of a parallel robot driven by cables relates the Cartesian velocity $\tau_{e}$ of the mobile platform to the time derivative $\mathbf{i}=\left(\begin{array}{lll}\dot{l}_{1} & \ldots & i_{k}\end{array}\right)^{T}$ of cable length vector. The Cartesian velocity of the mobile platform expressed in a fixed reference $\mathcal{F}_{f}$ is ${ }^{f} \boldsymbol{\tau}_{e}={ }^{f} \boldsymbol{\tau}_{e / f}=$ $\left({ }^{f} \mathbf{V}_{e / f}{ }^{f} \boldsymbol{\Omega}_{e / f}\right)^{T}$.

The time derivative of (7) provides:

$$
\dot{l}_{i}=\mathbf{N}_{i}\left(\begin{array}{c}
{ }^{A i} \dot{\mathbf{B}}_{i} \\
\dot{L}_{i} \\
{ }^{A i} \dot{\vartheta}_{B i x}
\end{array}\right)
$$

where

$$
\begin{aligned}
& \text { - } \mathbf{N}_{i}=\left(\begin{array}{lllll}
N_{i 1} & 0 & N_{i 2} & N_{i 3} & N_{i 4}
\end{array}\right) \\
& \text { - } N_{i 1}=\frac{l_{i} r_{i}-\left(c_{1 i}-c_{2 i}\right)^{A i} B_{i z}}{r_{i} A i_{i x}}, N_{i 2}=\frac{c_{1 i}-c_{2 i}}{r_{i}}, N_{i 3}= \\
& \\
& \frac{-2 l_{i}+\left(c_{1 i}+c_{2 i}\right)^{A i} B_{i x}}{2 L_{i}} \text { and } N_{i 4}=\frac{2 l_{i}-\left(c_{1 i}+c_{2 i}\right)^{A i} B_{i x}}{2^{A i} \vartheta_{B i x}}
\end{aligned}
$$

The terms ${ }^{A i} \dot{\mathbf{B}}_{i}, \dot{L}_{i}$ and ${ }^{A i} \dot{\vartheta}_{B i x}$ in (8) can be expressed as the product of their associated interaction matrices $\mathbf{D}_{B i}, \mathbf{D}_{L i}$ and $\mathbf{D}_{s i}$ by ${ }^{f} \boldsymbol{\tau}_{e}$, so that (8) becomes:

$$
i_{i}=\mathbf{D}_{i}{ }^{f} \boldsymbol{\tau}_{e}
$$

where $\mathbf{D}_{i}=\mathbf{N}_{i}\left(\begin{array}{c}\mathbf{D}_{B i} \\ \mathbf{D}_{L i} \\ \mathbf{D}_{s i}\end{array}\right)$, the expression of $\mathbf{D}_{B i}, \mathbf{D}_{L i}$ and $\mathbf{D}_{s i}$ being derived in the next subsections.

In the case of an inextensible cable, time derivative of the motorized joint angle vector $\mathbf{q}$ of the robot is linearly related to the time derivative of the cable length vector and can be computed as $\dot{\mathbf{q}}=\frac{1}{r_{c}} \dot{\mathrm{i}}$, where $r_{c}$ is the radius of the drums collecting the cables. Therefore, the instantaneous inverse kinematic model associated with the motorized joint angle vector $\mathbf{q}$ can be written as:

$$
\dot{\mathbf{q}}=\frac{1}{r_{c}} \mathbf{D}_{a}{ }^{f} \boldsymbol{\tau}_{e}
$$

where $\mathbf{D}_{a}$ is the compound matrix containing the matrices $\mathbf{D}_{i}, i=1 \ldots k$.

\section{A. Expression of $\mathbf{D}_{L i}$}

According, e.g., to [2], [11], the expressions of the time derivatives of $L_{i}$ and ${ }^{f} \underline{\mathbf{u}}_{i}$ (case of an inextensible and massless cable) are:

$$
\dot{L}_{i}=\mathbf{D}_{L i}{ }^{f} \boldsymbol{\tau}_{e}
$$

where $\mathbf{D}_{L i}={ }^{f} \underline{\mathbf{u}}_{i}^{T}\left(\mathbf{I}_{3} \quad-\left[{ }^{f} \mathbf{R}_{e}{ }^{e} \mathbf{B}_{i}\right]_{\times}\right)$, and:

$$
{ }^{f} \dot{\underline{\mathbf{u}}}_{i}=\mathbf{D}_{u i}{ }^{f} \boldsymbol{\tau}_{e}
$$

where $\mathbf{D}_{u i}=\frac{1}{L i}\left(\mathbf{I}_{3}-{ }^{f} \underline{\mathbf{u}}_{i}{ }^{f} \underline{\mathbf{u}}_{i}^{T}\right)\left(\mathbf{I}_{3}-\left[{ }^{f} \mathbf{R}_{e}{ }^{e} \mathbf{B}_{i}\right]_{\times}\right)$.

B. Expression of $\mathbf{D}_{B i}$

1) Angular velocity of $\mathcal{F}_{A i}$ expressed in $\mathcal{F}_{f}$ :

The time derivative of (1) gives:

$$
\dot{\gamma}_{i}=\mathbf{D}_{0 i}{ }^{b} \underline{\dot{\mathbf{u}}}_{i}=\mathbf{D}_{0 i}{ }^{b} \mathbf{R}_{f}{ }^{f} \underline{\dot{\mathbf{u}}}_{i}
$$

where $\mathbf{D}_{0 i}=\frac{1}{{ }^{b} u_{i x}^{2}+{ }^{b} u_{i y}^{2}}\left(\begin{array}{lll}-{ }^{b} u_{i y} & { }^{b} u_{i x} & 0\end{array}\right)$.

Taking into account the fact that ${ }^{f} \underline{\mathbf{z}}_{b}={ }^{f} \underline{\mathbf{Z}}_{A i}$ and according to (12), the angular velocity of $\mathcal{F}_{A i}$ is defined by:

$$
{ }^{f} \boldsymbol{\Omega}_{A i}=\mathbf{D}_{\gamma_{i}}{ }^{f} \boldsymbol{\tau}_{e}
$$

where $\mathbf{D}_{\gamma_{i}}={ }^{f} \underline{\mathbf{z}}_{A i} \mathbf{D}_{0 i}{ }^{b} \mathbf{R}_{f} \mathbf{D}_{u i}$.

2) Inverse kinematic model associated with ${ }^{A i} \dot{\mathbf{B}}_{i}$ :

The time derivative of $\mathbf{B}_{i}$, with respect to the fixed reference frame $\mathcal{F}_{f}$, is:

$$
{ }^{f} \dot{\mathbf{B}}_{i}=\mathbf{D}_{B f i}{ }^{f} \boldsymbol{\tau}_{e}
$$

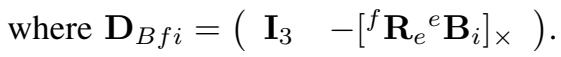

In the local frame $\mathcal{F}_{A i}$, the velocity of ${ }^{A i} \mathbf{B}_{i}$ has the following form:

$$
{ }^{A i} \dot{\mathbf{B}}_{i}={ }^{A i} \mathbf{R}_{f}\left[{ }^{f} \overrightarrow{\mathbf{A}_{i} \mathbf{B}_{i}}\right] \times{ }^{f} \boldsymbol{\Omega}_{A i}+{ }^{A i} \mathbf{R}_{f}{ }^{f} \dot{\mathbf{B}}_{i}
$$

Substituting (14) and (15) in (16) gives:

$$
{ }^{A i} \dot{\mathbf{B}}_{i}=\mathbf{D}_{B i}{ }^{f} \boldsymbol{\tau}_{e}
$$

where $\mathbf{D}_{B i}={ }^{A i} \mathbf{R}_{f}\left(\left[{ }^{f} \overrightarrow{\mathbf{A}_{i} \mathbf{B}_{i}}\right]_{\times} \mathbf{D}_{\gamma_{i}}+\mathbf{D}_{B f i}\right)$.

\section{Expression of $\mathbf{D}_{s i}$}

1) Mobile platform static equilibrium:

One can write the force applied at point $\mathbf{B}_{i}$ by the cable to the platform as (see Eq. (6)):

$$
{ }^{f} \mathbf{f}_{B i}=-{ }^{f} \boldsymbol{\vartheta}_{B i}=-{ }^{f} \mathbf{u}_{B i}
$$

where $\vartheta_{B i} \geq 0$ since a cable can only pull on the platform.

Assuming that the moment of ${ }^{f} \mathbf{f}_{B i}$ at point $\mathbf{B}_{i}$ is $\boldsymbol{\eta}_{B i}=$ $\mathbf{0}$, the wrench applied by cable $i$ at the reference point $\mathbf{E}$, expressed in $\mathcal{F}_{f}$, is:

$$
\left.{ }^{f}\right\urcorner_{i}=\left(\begin{array}{c}
{ }_{\mathbf{f}_{B i}} \\
{ }_{{ }^{f} \overrightarrow{\mathbf{E B}_{i} \times{ }^{f}} \mathbf{f}_{B i}}
\end{array}\right)
$$

According to the mobile platform equilibrium, one can write the relationship between the external wrenches $f\urcorner_{F e}$ and cable wrenches as:

$$
\left.f\urcorner_{F e}+\sum_{i=1}^{k}\left({ }^{f}\right\urcorner_{i}\right)=\mathbf{0}
$$

Assuming that $f \dot{ך}_{F e}=\mathbf{0}$ (a pseudo-static case), the time derivative of (20) leads to:

$$
\sum_{i=1}^{k}\left({ }^{f} \dot{\rightarrow}_{i}\right)=\mathbf{0}
$$


where

$$
{ }_{f} \dot{ך}_{i}=-\left(\begin{array}{c}
{ }^{f} \dot{\mathbf{u}}_{B i} \\
\underset{{ }_{f}}{\overrightarrow{\mathbf{E} B_{i}} \times{ }^{f}} \mathbf{u}_{B i}+{ }^{f} \overrightarrow{\mathbf{E B}_{i}} \times{ }^{f} \dot{\mathbf{u}}_{B i}
\end{array}\right)
$$

At this time, we can write:

$$
{ }_{f} \dot{\overrightarrow{\mathbf{E B}_{i}}}=\mathbf{D}_{E B i}{ }^{f} \boldsymbol{\tau}_{e}
$$

where $\mathbf{D}_{E B i}=\left(\begin{array}{ll}\mathbf{0}_{3} & -\left[{ }^{f} \mathbf{R}_{e}{ }^{e} \mathbf{B}_{i}\right]_{\times}\end{array}\right)$.

Therefore (22) becomes:

$$
\dot{\boldsymbol{خ}}_{i}=-\left(\begin{array}{c}
{ }^{f} \dot{\mathbf{u}}_{B i} \\
-\left[{ }^{f} \mathbf{u}_{B i}\right]_{\times} \mathbf{D}_{E B i}{ }^{f} \boldsymbol{\tau}_{e}+\left[{ }^{f} \overrightarrow{\mathbf{E B}}\right]_{i}{ }^{f} \dot{\mathbf{u}}_{B i}
\end{array}\right)
$$

2) Time derivative of ${ }^{f} \mathbf{u}_{B i}$ :

The time derivative of (6), expressed in the fixed frame $\mathcal{F}_{f}$, gives:

$$
{ }^{f} \dot{\mathbf{u}}_{B i}={ }^{f} \dot{\mathbf{u}}_{i}+{ }^{f} \dot{\mathbf{u}}_{\rho_{i}}
$$

Using (3), the time derivative of $\beta_{0 i}$ can be computed as:

$$
\dot{\beta}_{0 i}=\frac{\cos \left(\beta_{0 i}\right)^{2}}{{ }^{A i} B_{i x}^{2}}\left(\begin{array}{lll}
-{ }^{A i} B_{i z} & 0 & { }^{A i} B_{i x}
\end{array}\right){ }^{A i} \dot{\mathbf{B}}_{i}
$$

Thus, according to (26) and to the expression of $\mathbf{u}_{i}$ given in Section II-A, the time derivative of ${ }^{f} \mathbf{u}_{i}$ is:

$$
{ }^{f} \dot{\mathbf{u}}_{i}=\mathbf{D}_{f i}{ }^{A i} \dot{\mathbf{B}}_{i}+\frac{{ }^{f} \underline{\mathbf{u}}_{i}}{\cos \left(\beta_{0 i}\right)}{ }^{A i} \dot{\vartheta}_{B i x}+\frac{{ }^{A i} \vartheta_{B i x}}{\cos \left(\beta_{0 i}\right)}{ }^{f} \dot{\underline{\mathbf{u}}}_{i}
$$

By substituting (12) and (17) in (27), one can deduce:

$$
{ }^{f} \dot{\mathbf{u}}_{i}=\mathbf{D}_{u i 1}{ }^{f} \boldsymbol{\tau}_{e}+\mathbf{D}_{u i 2}{ }^{A i} \dot{\vartheta}_{B i x}
$$

where

$$
\begin{aligned}
& \text { - } \mathbf{D}_{u i 1}=\left(\mathbf{D}_{f i} \mathbf{D}_{B i}+\frac{{ }^{A i} \vartheta_{B i x}}{\cos \left(\beta_{0 i}\right)} \mathbf{D}_{u i}\right) \\
& \text { - } \mathbf{D}_{f i}=\frac{{ }^{A i} \vartheta_{B i x} \sin \left(\beta_{0 i}\right)^{f} \underline{\underline{\mathbf{u}}}_{i}}{{ }^{A i} B_{i x}^{2}}\left(\begin{array}{lll}
-{ }^{A i} B_{i z} & 0 & { }^{A i} B_{i x}
\end{array}\right) \\
& \text { - } \mathbf{D}_{u i 2}=\frac{{ }^{f} \underline{\underline{u}}_{i}}{\cos \left(\beta_{0 i}\right)}
\end{aligned}
$$

Then, using (11), the time derivative of ${ }^{f} \mathbf{u}_{\rho_{i}}=\frac{\rho_{0} g L_{i} f}{2} \underline{\mathbf{z}}_{A i}$ is:

$$
{ }^{f} \dot{\mathbf{u}}_{\rho_{i}}=\mathbf{D}_{u \rho_{i}}{ }^{f} \boldsymbol{\tau}_{e}
$$

where $\mathbf{D}_{u \rho_{i}}=\frac{\rho_{0} g}{2}{ }^{\prime} \underline{\mathbf{z}}_{A i} \mathbf{D}_{L i}$.

Finally, the time derivative of the non-unit vector ${ }^{f} \mathbf{u}_{B i}$ is:

$$
{ }^{f} \dot{\mathbf{u}}_{B i}=\left(\mathbf{D}_{u i 1}+\mathbf{D}_{u \rho_{i}}\right)^{f} \boldsymbol{\tau}_{e}+\mathbf{D}_{u i 2}{ }^{A i} \dot{\vartheta}_{B i x}
$$

3) Expression of ${ }^{A i} \dot{\vartheta}_{B i x}$ :

Using (30) and (24), one can deduce:

$$
f \dot{\boldsymbol{T}}_{i}=-\left(\mathbf{D}_{\tau_{i}}{ }^{f} \boldsymbol{\tau}_{e}+\mathbf{D}_{\vartheta_{i}}{ }^{A i} \dot{\vartheta}_{B i x}\right)
$$

where

$$
\begin{aligned}
& \text { - } \mathbf{D}_{\tau_{i}}=\left(\begin{array}{c}
\mathbf{D}_{u \rho_{i}}+\mathbf{D}_{u i 1} \\
\left.-{ }^{f} \mathbf{u}_{B i}\right]_{\times} \mathbf{D}_{E B i}+\left[{ }^{f} \overrightarrow{\mathbf{E} \mathbf{B}_{i}}\right]_{\times}\left(\mathbf{D}_{u \rho_{i}}+\mathbf{D}_{u i 1}\right)
\end{array}\right) \\
& \text { - } \mathbf{D}_{\vartheta_{i}}=\left(\begin{array}{c}
\mathbf{D}_{u i 2} \\
\left.{ }_{\left[{ }^{f}\right.} \overrightarrow{\mathbf{E} \mathbf{B}_{i}}\right]_{\times} \mathbf{D}_{u i 2}
\end{array}\right)
\end{aligned}
$$

Eq. (21) becomes:

$$
\left(\sum_{i=1}^{k} \mathbf{D}_{\tau_{i}}\right)^{f} \boldsymbol{\tau}_{e}+\mathbf{D}_{\vartheta}{ }^{A i} \dot{\boldsymbol{\vartheta}}_{B x}=\mathbf{0}
$$

where
- $\mathbf{D}_{\vartheta}=\left(\mathbf{D}_{\vartheta_{1}}, \ldots, \mathbf{D}_{\vartheta_{k}}\right)$

- ${ }^{A i} \dot{\boldsymbol{\vartheta}}_{B x}=\left({ }^{A i} \dot{\vartheta}_{B 1 x}, \ldots,{ }^{A i} \dot{\vartheta}_{B k x}\right)^{T}$

Finally, one can compute ${ }^{A i} \dot{\boldsymbol{\vartheta}}_{B x}$ as:

$$
{ }^{A i} \dot{\boldsymbol{\vartheta}}_{B x}=\mathbf{D}_{s}{ }^{f} \boldsymbol{\tau}_{e}
$$

where $\mathbf{D}_{s}=-\mathbf{D}_{\vartheta}^{+} \sum_{i=1}^{k} \mathbf{D}_{\tau_{i}}$. Let us note that the use of the pseudo-inverse to compute $\mathbf{D}_{s}$ may not be the best choice. This potential issue is however out of the scope of this paper

Hence, ${ }^{A i} \dot{\vartheta}_{B i x}$ can be found by:

$$
{ }^{A i} \dot{\vartheta}_{B i x}=\mathbf{D}_{s i}{ }^{f} \boldsymbol{\tau}_{e}
$$

where $\mathbf{D}_{s i}$ is the ith row of matrix $\mathbf{D}_{s}$.

\section{VISION-BASED CONTROL}

\section{A. Control}

Visual servoing is based on the so-called interaction matrix $\mathbf{L}_{\mathbf{s}}$ which relates the instantaneous relative Cartesian motion $\tau$ between the mobile platform and the scene to the time derivative of the vector $\mathbf{s}$ of the visual primitives used for regulation [17] $\left(\dot{\mathbf{s}}=\mathbf{L}_{\mathbf{s}} \boldsymbol{\tau}\right)$. According to the nature of the visual primitives, there exist many visual servoing techniques ranging from position-based visual servoing [18], [19] to image-based visual servoing [4], [20], most of them being based on point features. One can also find other visual primitives such as lines [21] or image moments [22].

As the instantaneous inverse kinematic model (10) depends on the platform pose, we choose position-based visual servoing in the 3D pose form [18], [19], [23]: $\mathbf{s}=$ $\left(\begin{array}{ll}\mathbf{s}_{t} & \mathbf{s}_{w}\end{array}\right)^{T}$.

Consider $\mathcal{F}_{m}$ and $\mathcal{F}_{m^{*}}$ the current and the desired mobile frame locations, respectively. $\mathbf{s}_{t}={ }^{m} \mathbf{t}_{m^{*}}$ is the translation error between $\mathcal{F}_{m}$ and $\mathcal{F}_{m^{*}}$ and $\mathbf{s}_{w}=\underline{\mathbf{u}} \theta$, where $\underline{\mathbf{u}}$ is the axis and $\theta$ is the angle of the rotation matrix ${ }^{m} \mathcal{R}_{m^{*}}$.

The interaction matrix associated to the pose can be written as [23], [24]:

$$
\mathbf{L}_{\mathbf{s}}=\left(\begin{array}{cc}
-\mathbf{I}_{3} & {\left[{ }^{m} \mathbf{t}_{m^{*}}\right]_{\times}} \\
\mathbf{0}_{3} & -\mathbf{L}_{w}
\end{array}\right)
$$

where

$$
\begin{aligned}
& \text { - } \mathbf{L}_{w}=\mathbf{I}_{3}-\frac{\theta}{2}[\underline{\mathbf{u}}]_{\times}+\left(1-\frac{\operatorname{sinc}(\theta)}{\operatorname{sinc}^{2}\left(\frac{\theta}{2}\right)}\right)[\underline{\mathbf{u}}]_{\times}^{2} \\
& \text { - } \operatorname{sinc}(\theta)=\frac{\sin (\theta)}{\theta} \text { and }{ }^{m} \boldsymbol{\tau}_{m}={ }^{m} \boldsymbol{\tau}_{m / m^{*}}={ }^{m} \boldsymbol{\tau}_{m / f}
\end{aligned}
$$

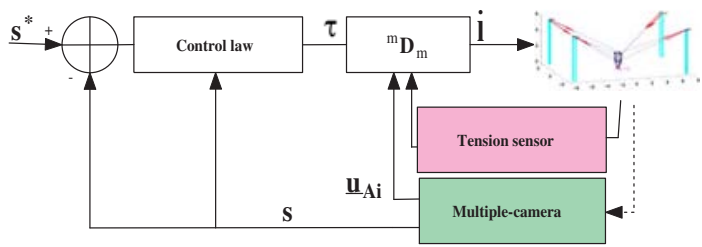

Fig. 3. Visual servoing of a cable-driven parallel robot

To regulate the error between the current primitive vector $\mathbf{s}$ and the desired one $\mathbf{s}^{*}=\mathbf{0}$, one can consider the exponential decay $\dot{\mathbf{s}}=-\lambda \mathbf{s}$. The vision-based control (Fig. 3) can then be expressed as:

$$
{ }^{m} \boldsymbol{\tau}_{m}=-\lambda \widehat{\mathbf{L}}_{\mathbf{s}}^{+} \mathbf{s}
$$


With ${ }^{f} \boldsymbol{\tau}_{e}=\mathbf{D}_{t}{ }^{m} \boldsymbol{\tau}_{m}$ and using (36), (10) becomes:

$$
\dot{\mathbf{q}}={ }^{m} \mathbf{D}_{m}{ }^{m} \boldsymbol{\tau}_{m}=-\lambda^{m} \mathbf{D}_{m}{\widehat{\mathbf{L}_{\mathbf{s}}}}^{+} \mathbf{s}
$$

where $\mathbf{D}_{t}=\left(\begin{array}{cc}{ }^{f} \mathbf{R}_{m} & { }^{f} \mathbf{R}_{m}\left[{ }^{m} \overrightarrow{\mathbf{E m}}\right]_{\times} \\ \mathbf{0}_{3} & { }^{f} \mathbf{R}_{m}\end{array}\right)$ and ${ }^{m} \mathbf{D}_{m}=\frac{1}{r_{c}} \mathbf{D}_{a} \mathbf{D}_{t}$.

B. Examples of measured variables and parameters to identify

Note that the instantaneous inverse kinematic model (10) depends on ${ }^{f} \mathbf{T}_{m}$, the rigid transformation between the mobile and the fixed frame which defines the pose of the platform. It depends also on the tangents ${ }^{f} \underline{\mathbf{u}}_{A i}$ to the cables at their drawing points, which we plan to measure by vision, and on some constant (calibration) parameters $\left({ }^{b} \mathbf{A}_{i},{ }^{e} \mathbf{B}_{i}\right.$, the transformations ${ }^{m} \mathbf{T}_{e}$ and ${ }^{f} \mathbf{T}_{b}$ ). Let us also note that only information from the vision sensor are used to define the interaction matrix given in (35), using ${ }^{m} \mathbf{T}_{m^{*}}={ }^{m} \mathbf{T}_{f}{ }^{f} \mathbf{T}_{m^{*}}$.

We plan to use 12 cameras. 4 cameras are used to measure the pattern position (one at the top of each post). Additionally, 4 stereo pairs should allow us to measure the tangent direction at the cable drawing points. This particular setup is not the only possible one but has been selected based on practical constraints. All kinematic parameters are expressed in a reference frame $\mathcal{F}_{f}=\mathcal{F}_{c j}$ attached to the base frame.

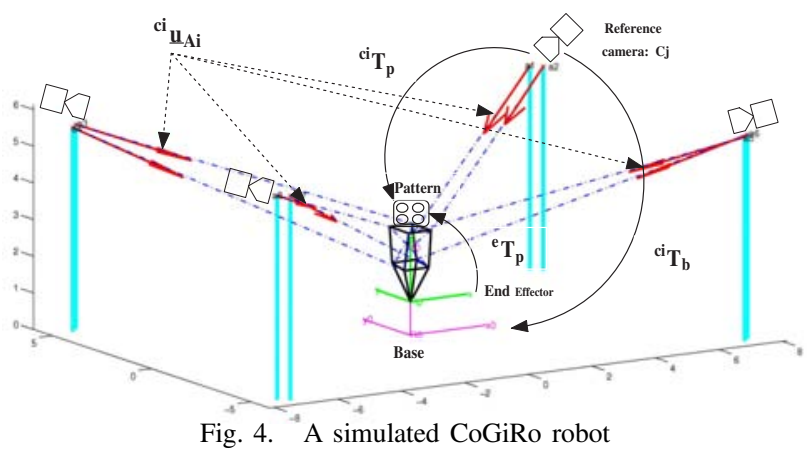

In the instantaneous inverse kinematic model (10), we need to define the rotation ${ }^{A i} \mathbf{R}_{b}$ which depends on the angle $\gamma_{i}$ as shown in (1). This angle can also be computed using $\gamma_{i}=\tan ^{-1}\left({ }^{b} u_{A i y}\right)$, where ${ }^{b} \underline{\mathbf{u}}_{A i}={ }^{b} \mathbf{R}_{f}{ }^{f} \underline{\mathbf{u}}_{A i}$. We need also the angle $\beta_{0 i}$ defined in (3). This angle can be computed using the following expressions of the attachment point $\mathbf{B}_{i}$ :

$$
\left\{\begin{array}{l}
{ }^{A i} \mathbf{B}_{i}={ }^{A i} \mathbf{R}_{b}\left({ }^{b} \mathbf{R}_{f}{ }^{f} \mathbf{B}_{i}+{ }^{b} \mathbf{t}_{f}\right)+{ }^{A i} \mathbf{t}_{b} \\
{ }^{f} \mathbf{B}_{i}={ }^{f} \mathbf{R}_{m}{ }^{m} \mathbf{B}_{i}+{ }^{f} \mathbf{t}_{m}
\end{array}\right.
$$

where ${ }^{f} \mathbf{R}_{m}$ and ${ }^{f} \mathbf{t}_{m}$ can be measured by vision and ${ }^{m} \mathbf{B}_{i}$ is a constant parameter.

The instantaneous inverse kinematic model (10) depends also on the length $L_{i}=\left\|f \overrightarrow{\mathbf{A}_{i} \mathbf{B}_{i}}\right\|$ of cable $i$ supposed to be massless (straight line) and on the component ${ }^{A i} \vartheta_{B i x}$ of the force applied to the cable at its attachment point $\mathbf{B}_{i}$.

Using (6), the non-unit vector ${ }^{f} \mathbf{u}_{B i}$ can be computed as:

$$
{ }^{f} \mathbf{u}_{B i}={ }^{f} \boldsymbol{\vartheta}_{B i}=-{ }^{A i} \vartheta_{A i}{ }^{f} \underline{\mathbf{u}}_{A i}+\rho_{0} g L_{i}{ }^{f} \underline{\mathbf{z}}_{A i}
$$

where ${ }^{f} \underline{\mathbf{z}}_{A i}={ }^{f} \mathbf{R}_{b}{ }^{b} \underline{\mathbf{z}}_{A i}$ and ${ }^{A i} \vartheta_{A i}$ is given by a tension sensor (possibly indirectly).

Consequently, using ${ }^{A i} \boldsymbol{\vartheta}_{B i}={ }^{A i} \mathbf{R}_{b}{ }^{b} \mathbf{R}_{f}{ }^{f} \boldsymbol{\vartheta}_{B i}$, one can compute ${ }^{A i} \vartheta_{B i x}={ }^{A i} \underline{\mathbf{x}}_{A i}^{T}{ }^{A i} \boldsymbol{\vartheta}_{B i}$.

\section{Simulation Results}

The vision-based control strategy introduced in the previous section is validated by means of a CoGiRo cabledriven parallel robot simulation (Fig. 4). CoGiRo is a 6-DOF large-dimension parallel cable-driven robot. It has a moving platform (end-effector) connected to a fixed base by 8 driving cables of varying lengths $l_{i}, i \in 1 \ldots 8(\mathrm{k}=8)$. Each cable (Fig. 4) is attached to the moving platform at point $\mathbf{B}_{i}$ and extends from the base at point $\mathbf{A}_{i}$.

In the simulation, ${ }^{b} \mathbf{E}=(0,0,0)^{T} m$ and $\left(\theta_{x}, \theta_{y}, \theta_{z}\right)^{T}=$ $(0,0,0)^{T}$ defines the initial configuration of the CoGiRo platform simulation. The desired one (Fig. 5) is ${ }^{b} \mathbf{E}^{*}=$ $(2,1,1)^{T} m$ and $\left(\theta_{x}^{*}, \theta_{y}^{*}, \theta_{z}^{*}\right)^{T}=\left(0,10^{\circ}, 20^{\circ}\right)^{T}$.

In a first simulation, we choose three geometric characteristic cases (dimensions: width $\mathrm{x}$ length $\mathrm{x}$ height) of the CoGiRo robot. Tab. II presents the angles $d \beta_{i}=\beta_{i}-\beta_{0 i}$ (See Fig. 2 for the definition of these angles), in the desired position. $d \beta_{i}$ increases according to the dimensions of the robot which confirms that the cables can not be considered as a straight lines segments, in the case of a large-dimension parallel cable-driven robots.

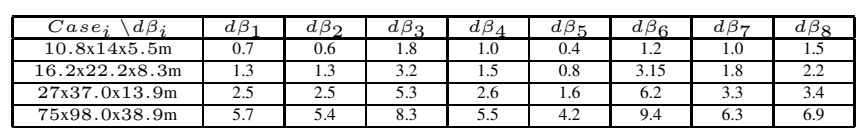

TABLE II

Evolution of $d \beta_{i}=\beta_{i}-\beta_{0 i}$ (DEGREES).

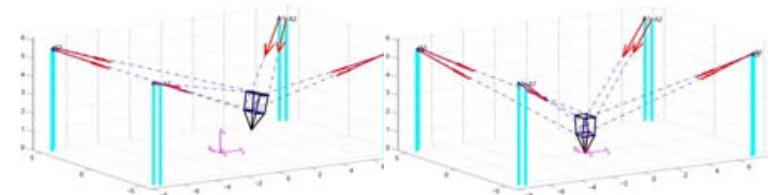

Fig. 5. Desired (left) and initial (right) position of the CoGiRo robot.

In a second simulation, we choose the dimensions of the real CoGiRo robot $(10.8 \times 14 \times 5.5 \mathrm{~m}$ (width $\mathrm{x}$ length $\mathrm{x}$ height)). To show the robustness of the proposed vision-based control, we added noise to the estimation of the Cartesian pose ${ }^{f} \mathbf{T}_{m}$. We define at each time a sample random noise for translation with maximal value of $5 \mathrm{~cm}$. We also added noise to the rotation angles, with amplitude of $2^{\circ}$. Assuming that the directions of the tangents to the cable curves at their drawing points is essentially a unit vector, the added noise can be modeled by a rotation of the unit vector in each direction. Consequently, we define at each time a sample random rotation axis and a positive rotation angle with maximal amplitude of $0.2^{\circ}$.

Fig. 6 shows a potentially good robustness. As expected, the Cartesian errors converge exponentially to final errors displayed in Table III, with a perfect decoupling. The 3D trajectory of the mobile platform (Fig. 7) is linear in the fixed reference frame $\mathcal{F}_{f}=\mathcal{F}_{c j}$, which confirms the properties of the $3 \mathrm{D}$ pose visual servoing.

Table III presents mean values and standard deviations of the final error vector norm. These accuracy results are deemed satisfactory enough for such a large-dimension parallel robot. 


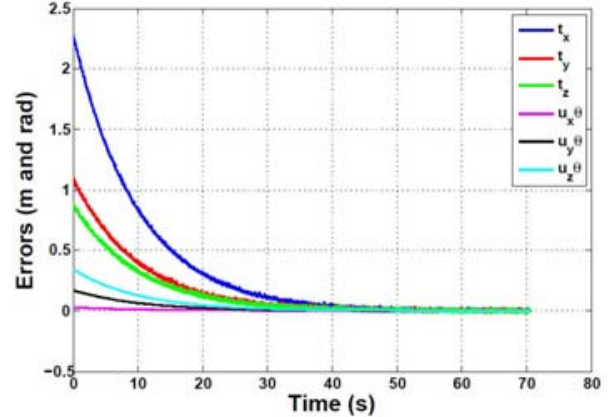

Fig. 6. Evolution of the Cartesian errors.

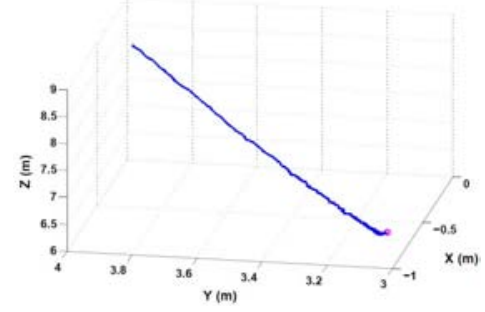

Fig. 7. Evolution of the mobile platform reference point trajectory.

\begin{tabular}{|c|c|c|}
\hline & Mean value & Standard deviation \\
\hline Translation error $(\mathrm{mm})$ & 8.2 & 6.5 \\
\hline Orientation error $\left(^{\circ}\right)$ & 0.051 & 0.032 \\
\hline
\end{tabular}

TABLE III

MEAN VALUES AND STANDARD DEVIATIONS OF FINAL CARTESIAN ERRORS

\section{CONCLUSION}

In this paper, we have introduced an instantaneous inverse kinematic model of large-dimension cable-driven parallel robots. This model relies on a simplified model of an inextensible hefty cable in which the cable profile is considered to be a parabolic curve. It can be used to compute an explicit expression of each cable tangent and to compute a partially decoupled inverse kinematic problem. We have shown that the proposed instantaneous inverse kinematic model depends on the mobile platform pose, the cable tangent directions and the cable tensions. A multi-camera setup should allow us to measure the pose of a visual target attached to the mobile platform and the direction of the cable tangents. The cable tensions are measured by means of force sensors. Computer vision can then be used in the feedback loop by using of a 3D pose kinematic visual servoing method. This latter has been validated on the simulator of a large parallel cable robot.

The next step in our work is thus to implement the visionbased control strategy proposed in this paper on a real parallel CoGiRo cable robot.

\section{ACKNOWLEDGMENT}

This work was supported by the ANR (grant 2009 SEGI 018, CoGiRo project).

\section{REFERENCES}

[1] R. G. Roberts, T. Graham, and T. Lippitt. On the inverse kinematics, statics, and fault tolerance of cable-suspended robots. Journal of Robotic Systems, 15:581-597, 1998.

[2] J.P. Merlet. Parallel robots. Kluwer Academic Publishers, 2000.
[3] L. Weiss, A. Sanderson, and C. Neuman. Dynamic sensor-based control of robots with visual feedback. In IEEE Trans. on Robotics and Automation, volume 3, pages 404-417, October 1987.

[4] B. Espiau, F. Chaumette, and P. Rives. A new approach to visual servoing in robotics. IEEE Trans. on Robotics and Automation, 8(3):313-326, June 1992.

[5] S. Hutchinson, G.D. Hager, and P.I. Corke. A tutorial on visual servo control. IEEE Transactions on Robotics and Automation, 12(5):651670, October 1996.

[6] M.L. Koreichi, S. Babaci, F. Chaumette, G. Fried, and J. Pontnau. Visual servo control of a parallel manipulator for assembly tasks. In 6th Int. Symposium on Intelligent Robotic Systems, SIRS'98, pages 109-116, Edimburg, Scotland, July 1998.

[7] P. Kallio, Q. Zhou, and H. N. Koivo. Three-dimensional position control of a parallel micromanipulator using visual servoing. In Bradley J. Nelson and Jean-Marc Breguet, editors, Microrobotics and Microassembly II, Proceedings of SPIE, volume 4194, pages 103-111, Boston USA, November 2000.

[8] T. Dallej, N. Andreff, Y. Mezouar, and P. Martinet. 3D pose visual servoing relieves parallel robot control from joint sensing. In Proceedings of the IEEE International Conference on Intelligent Robots and Systems, IROS'06, pages 4291-4296, Beijing, China, October 2006.

[9] N. Andreff, T. Dallej, and P. Martinet. Image-based visual servoing of Gough-Stewart parallel manipulators using legs observation. Joint Issue of IJCV and IJRR on Vision and Robotics, 26(7):677-687, 2007.

[10] T. Dallej, N. Andreff, and P. Martinet. Towards a generic imagebased visual servoing of parallel robots using legs observation. In Proceedings of the World Congress in Mechanism and Machine Science, IFToMM07, Besançon, France, June 2007.

[11] T. Dallej, M. Gouttefarde, N. Andreff, M. Michelin, and P. Martinet. Towards vision-based control of cable-driven parallel robots. In IEEE International Conference on Intelligent Robots and Systems, IROS, pages 2855-2860, San Francisco, California, September 2011.

[12] N. Riehl, M. Gouttefarde, C. Baradat, and F. Pierrot. On the determination of cable characteristics for large dimension cable-driven parallel mechanisms. In IEEE Transactions on Robotics and Automation, pages 4709 - 4714, Alaska, USA, May 2010.

[13] H. Irvine. Cable Structures. MA: MIT Press, Cambridge, 1981.

[14] K. Kozak, Q. Zhou, and J. Wang. Static analysis of cable-driven manipulators with non-negligible cable mass. IEEE Transactions on Robotics, 22(3):425-433, 2006.

[15] M. Gouttefarde, J.-F. Collard, N. Riehl, and C. Baradat. Simplified static analysis of large-dimension parallel cable-driven robots. In IEEE International Conference on Robotics and Automation, ICRA, St Paul, Minnesota, May 14-18 2012.

[16] E. Malis, François Chaumette, and S. Boudet. Multi-cameras visual servoing. In IEEE Int. Conf. on Robotics and Automation, ICRA'OO, volume 4, pages 3183-3188, San Francisco, États-Unis, 2000.

[17] C. Samson, M. Leborgne, and B. Espiau. Robot Control: The Task Function approach. Oxford Engineering Series, 22, Oxford University Press, ISBN-13: 978-0198538059, 1991.

[18] W. J. Wilson, C. C. Williams Hulls, and G. S. Bell. Relative endeffector control using cartesian position-based visual servoing. IEEE Transactions on Robotics and Automation, 12(5):684-696, 1996.

[19] P. Martinet, N. Daucher, J. Gallice, and M. Dhome. Robot control using 3D monocular pose estimation. In Proceedings of the Workshop on New Trends in Image Based Robot Servoing, IEEE/RSJ International Conference on Intelligent Robots and Systems, IROS'97, pages 1-12, Grenoble, France, September 1997.

[20] S. Benhimane and E. Malis. Homography-based 2D visual servoing. In IEEE International Conference on Robotics and Automation, pages 2397-2402, Orlando, USA, May 2006.

[21] N. Andreff, B. Espiau, and R. Horaud. Visual servoing from lines. Int. Journal of Robotics Research, 21(8):679-700, August 2002.

[22] O. Tahri and F. Chaumette. Application of moment invariants to visual servoing. In IEEE International Conference on Robotics and Automation, volume 3, pages 4276-4281, Taiwan, May 2003.

[23] E. Malis, F. Chaumette, and S. Boudet. 2 1/2 d visual servoing. In IEEE International Conference on Robotics and Automation, volume 15, pages 238-250, Detroit, USA, April 1999.

[24] B Thuilot, P. Martinet, L Cordesses, and J. Gallice. Position based visual servoing: keeping the object in the field of vision. In IEEE International Conference on Robotics and Automation, pages 16241629, Washington, USA, May 2002. 